\title{
Nail Surface Topography and Onychochronobiology
}

\author{
Gérald E. Piérard and Claudine Piérard-Franchimont
}

\subsection{Introduction}

Some chronic skin disorders are responsible for concurrent nail changes. Typical examples are represented by psoriasis, lichen planus, and alopecia areata. The lifetime incidence of nail alterations in these patients potentially reaches $80 \%$ or over. However, the nail involvement remains frequently overlooked by physicians, despite the burden to the patients caused by functional impairment of manual dexterity, pain, and psychological stress [1].

The nail plate is hard but flexible. It grows continuously and is frequently subject to a number of microtraumatisms. A healthy nail plate grossly looks smooth, but closer examination reveals it is not the case. Its specific surface microrelief has physiologic and sometimes pathologic implications. At close inspection, the regular relief is fine and only visible in children. In middle-aged individuals, the physiologic changes often remain too discrete to be readily discernable. By contrast, it is noticeable with the naked eye in a number of elderly people.

G.E. Piérard, MD, PhD $(\bowtie)$

C. Piérard-Franchimont

Laboratory of Skin exhibit and Imaging,

Department of Dermatopathology,

University Hospital of Liège,

CHU SartTilman, Liège B-4000, Belgium

e-mail: gerald.pierard@ulg.ac.be
Several distinctive patterns of nail surface changes are clearly identified and distinguished by clinical inspection. However, the structure of the nail surface has attracted little attention from researchers. Descriptive reports are rarely supported by quantification of the nail plate microrelief $[2,3]$. Nail alterations result from any underlying chronic dermatoses and/or from external trauma and weathering. To analyze the nail relief, it is important to have at one's disposal reliable clinical or instrumental methods identifying minimal alterations and providing qualitative and quantitative data.

\subsection{Clinical Descriptive Approach}

Several typical nail changes are distinguished. Their combination is used to derive a nail severity index such as in psoriasis [2]. In this condition, affected nail plates are often thick and crumble. Because the lesions are obviously visible, patients are concerned about the appearance of their nails, often causing them to avoid normal daily activities including work. In the longer term, nail involvement is possibly a signal of a more severe form of the disease $[4,5]$.

The nail psoriasis severity index (NAPSI) was developed as an objective and reproducible tool for estimating nail involvement [2]. NAPSI was claimed to be reproducible and easy to handle. Indeed, it represents an improvement in rating 
treatment efficacy in nail psoriasis. For this purpose, each nail is divided into four quadrants to be assessed for the presence of any psoriatic change originating from both the nail matrix (pitting, leukonychia, nail plate crumbling) and the nail bed (oil drop discoloration, onycholysis, hyperkeratosis, splinter hemorrhages). If any sign is present in all four quadrants, the nail is given a score of 4 through to a score of 0 in absence of alteration in any quadrant. Each nail is assigned both a nail matrix and a nail bed score of $0-4$, which are combined to yield a total score of 0-8 for each nail. All nails are assessed with the total NAPSI score corresponding to the sum of the scores, reaching up to 80 if only the 10 finger nails are considered or up to 160 if toe nails are included. In practice, it is possible to target one single specific nail for assessing drug effects.

In medical practice, some drugs potentially alter the nail plates. For instance, retinoids commonly produce thinning and brittleness of the nail. Transverse ridging, white banding, as well as deep Beau's lines occasionally develop. Cancer chemotherapy also affects the nails. Multiple transverse white bands develop following cyclic chemotherapy. High-dose chemotherapy is responsible for Beau's lines sometimes followed by nail loss. Half-and-half nails, where the proximal part of the nail is whitish while the distal part is brownish, are not uncommon complication of cancer chemotherapy.

The typical appearance of onychomycosis presents as subungual hyperkeratosis with thickening and brownish discoloration of the nail plate. The nail plate becomes porous and crumble and it easily breaks off. In some patients, it is difficult to distinguish psoriatic onychodystrophy from fungal nail infection, but the recognition of the characteristic psoriatic onycholysis with a red-yellow halo and the so-called oil drops is helpful.

The twenty-nail dystrophy is a rather troublesome disease altering all or almost all the fingerand toe nails in children and adolescents. It combines excessive ridging of the nail plate, rough nails, and trachyonychia.

\subsection{Patterns of Nail Topography}

\subsubsection{Longitudinal Striations}

Longitudinal striations of the nail surface combine parallel grooves pulled apart by discrete projecting ridges. Such a condition is considered to be a physiologic feature when presenting as shallow grooves, usually parallel, and edged by low projecting ridges. Ridging commonly appears more prominent with aging and in association with some particular disorders.

Onychorrhexis consists of a series of narrow, longitudinal, parallel superficial striations. The nail appears as having been scratched by an awl. In some instances, dust material is ingrained into the superficial nail plate. The small linear projections extend from the proximal nail fold to the distal nail edge. Splitting of the free edge of the nail commonly ensues. In other instances, the nail ridging stops short or is interrupted at regular intervals, giving rise to a beaded appearance. In some patients a single wide longitudinal median ridge appears in cross section as a circumflex accent.

Median nail dystrophy is another uncommon condition of the thumb nails consisting of a longitudinal groove developed in the midline or just off center, starting at the cuticle and growing out of the free edge.

Any tumor located nearby the nail matrix exerts pressure and produces a single wide and deep longitudinal groove. This aspect vanishes when the tumor is removed.

\subsubsection{Herringbone Nail}

Oblique nail ridging, pointing centrally to meet in the midline, is an uncommon pattern particularly occurring during childhood.

\subsubsection{Beau's Lines}

Beau's lines correspond to transverse grooves extending between the lateral edges of the nail. 
They commonly affect all nails at similar levels. The width of the transverse groove is thought to be related to the duration of the causal process downgrading the nail matrix activity for a limited period of time. Any abrupt distal limit of the groove suggests a sudden outbreak of disease. By contrast, a sloping aspect suggests a progressive onset. The proximal limit of the depression is similarly abrupt or sloped according to the rate of normalization of the nail biology.

\subsubsection{Pitting and Rippling}

Rosenau's depressions refer to nail pitting and rippling. Pits develop as a result of defective nail formation in punctuate areas focused in the proximal portion of the nail matrix. The nail plate surface is studded in a buckshot pattern with punctuate hollow depressions varying in number, size, depth, and shape. An arbitrary figure of five pits is considered as a physiologic condition. The depth and width of the pits relate to the extent of the matrix involvement. Their length is determined by the duration of the nail damage. Deep and irregularly shaped pits often suggest psoriasis, but they are not specific for any disease including alopecia areata and other conditions.

Pits are randomly dispersed or evenly patterned in series along one or several longitudinal lines. They are sometimes aligned in a crisscross pattern resembling the external surface of a thimble. Regular pitting commonly leads to rippling or ridging.

\subsubsection{Trachyonychia}

Trachyonychia refers to a spectrum of alterations resulting in severe nail roughness as if the surface had been rubbed with sandpaper.

\subsubsection{Nail Underface Ridging}

The underface of the nail plate disclosed after avulsion exhibits topographic aspects unrelated to the outer surface of the same nail. Deep longitudinal striations are present and they commonly deepen with age.

\subsection{Bioinstrumentation}

The various metrology designs for the nail plate encompass routine methods as well as more sophisticated and experimental procedures. The examination of nail clippings is occasionally informative. The distinctive features of the nail microrelief are explored using various in vivo procedures. Clinical examination allows qualitative assessment of the gross surface topography. Low-magnification photographs under carefully controlled and repeatable conditions are conveniently used to document the nail surface microrelief. Transillumination helps disclosing some of the onychodystrophies. The method is rapid, simple, and cheap. The examination is performed in a dark environment. Digit transillumination is possible by shining a strong penlight beam upward through the pulp or alternatively by using the fiber optic from a high-power light device.

Controlled abrasion, microindentation, and sclerometry are used to investigate some physical properties of the nail surface. In particular, simulation of the worn nail by abrasion allows to assess the wear process, which is the prime target of cosmetic application of nail hardeners and nail varnishes. Sclerometry simulates by scrapping the effects of wear and tear on the nail surface. Such insults deteriorate the morphologic, mechanical, and optical properties and, thereby, the loss of the main properties of the nail.

The examination of the nail surface roughness can be performed following several noninvasive methods. High-resolution digital photography reveals some specific nail surface physical properties. The use of light filters selecting only a narrow wavelength spectrum contributes to the optical properties of the nail surface. Using polarized light reduces the glossy reflectance from the surface, thus improving visualization of 
fine nail dystrophies. Video microscopy is another noncontact method, helpful in revealing quantitative changes in the nail microrelief. Fringe projection is another method disclosing three-dimensional (3-D) nail surface contours.

The 3-D microscopy represents an advance in the analysis of the nail surface topography. Initially, the method aimed at controlling the quality of manufactured surfaces by assessing their roughness in a given direction. The parameters were standardized internationally. However, this method was limited to a two-dimensional (2-D) analysis of the surface structure with a signal representing roughness on a line of relief but with absence of a field image. This method was greatly improved by producing a record of a 3-D image of the surface. This achievement provides reconstructed 3-D images from an atomic scale (atomic force microscope) to macroscopic relief.

Scanning microdensitometry discriminates shadows and highlights photographic negatives of the nail surface taken under controlled procedures (light intensity, exposure time, angle of camera recording, and distance to the surface).

A technical advance was achieved with the introduction of negative replicas. Precise quantitative examinations are conveniently made in vivo on the outer portion of the nail. Alternatively, observations are performed after its avulsion or after making a negative replica. Scanning electron microscopy provides images of an unsurpassed morphologic accuracy. The examination of silicone replicas of the nail microrelief is an optional procedure. The microrelief of the nail replicas can be scrutinized using one of several methods including mechanical profilometry, autofocus laser beam profilometry, oblique illumination of the replicas, and optical measurement of the translucent replica thickness. The choice of the replica material is of importance. It has to polymerize quickly at the nail temperature, be adequately liquid to fill in all the erratic surface aspects, avoid deformation during polymerization, and avoid any artifact. The replica relief is scrutinized using a scanning tactile or optical analyzer.
The stringent use of optical profilometry or any other microtopographic assessment on nails or their replicas brings quantitative information [6]. The diversity of the typical alterations is clearly evidenced [7-10]. In addition, due to the sustained nail growth, onychochronobiology is explored using the same means at different time intervals [11, 12]. In addition, these methods are suitable for assessing the nail mechanical properties in combination with the microindentation and the sclerometry methods.

\subsubsection{Static Microtopography of the Nail Surface}

Quantitative assessment of the nail microtopography is usually performed on longitudinal scans [13]. Transverse scans are more difficult to interpret due to the natural nail curvature. When information has to be gained in this direction, it is recommended to examine sections no longer than $5 \mathrm{~mm}$ in length to minimize this pitfall. It should be kept in mind that native alterations such as shallow grooves and Beau's lines are better revealed at the proximal part of the nail (Fig. 40.1). Weathering and natural microabrasions add their effects in a cumulative way when moving toward the distal part of the nail (Figs. 40.2 and 40.3). Sources of variability such as uncontrolled nail plate wear should be discarded when appropriate. Controlled positioning of the nail is of the upmost importance for comparative purposes.

\subsubsection{Dynamic Microtopography of the Nail Surface}

Repeat controlled assessments overtime provide insight in onychochronobiology. By this way, the effects of therapies can be assessed. The speed of nail growth is conveniently recorded following the move of an engraved mark made at the initial examination. The linear nail growth is indeed measured following a shallow scratch in the proximal nail with a piece of hacksaw blade [14]. The nail mark is made at a defined distance (approximately 
Fig.40.1 Nail surface topography on longitudinal scans showing transverse grooves during cancer chemotherapy. (a) Twodimensional field image of a nail surface recording.

(b) Profilometric presentation
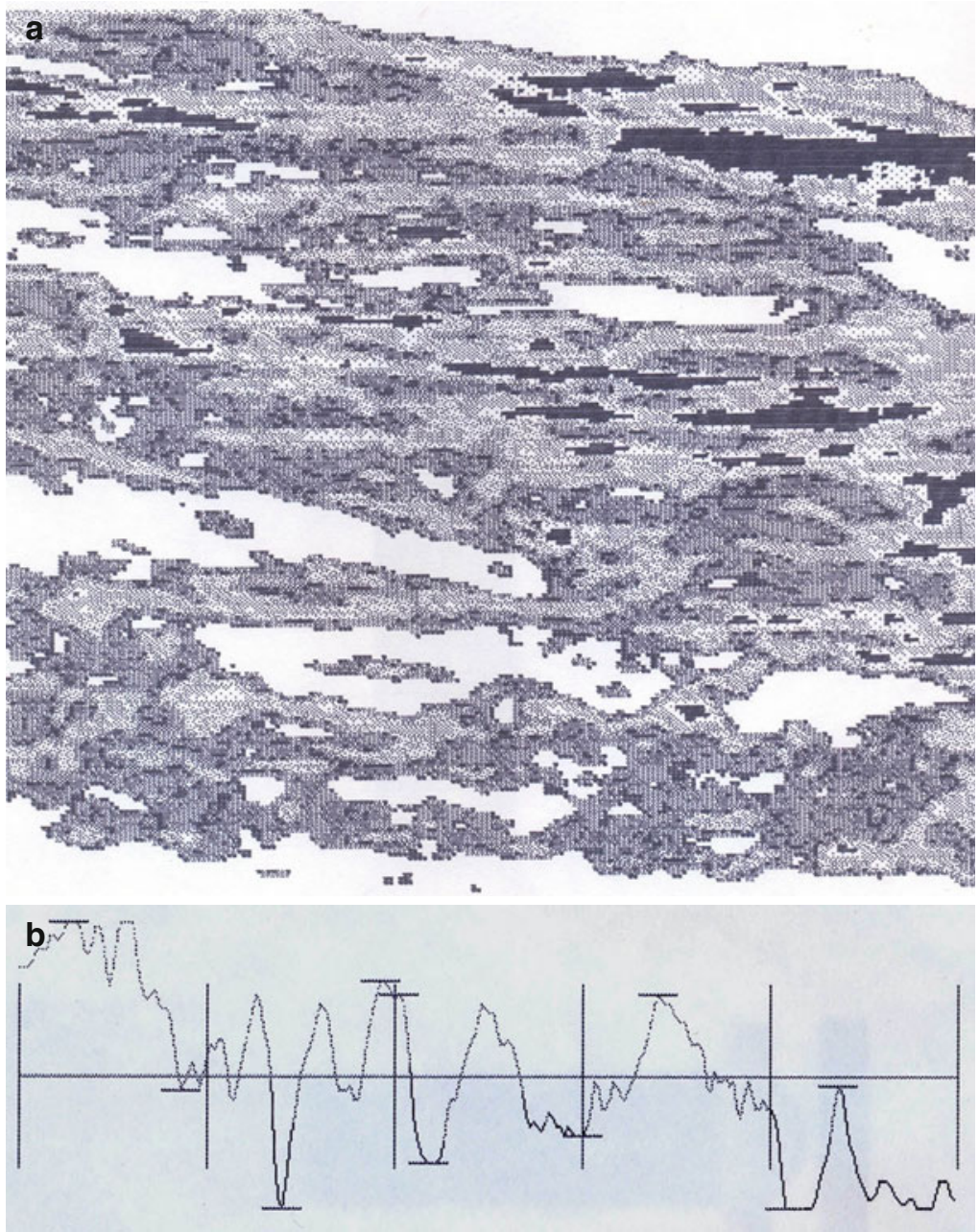

$3 \mathrm{~mm}$ ) from the proximal nail fold [15]. The subjects should refrain from pushing back their nail cuticles during the study period. After a period of approximately 1 month, the scratch has moved with the nail growth, and its distance to the proximal nail fold has increased. The average outgrowth is divided by the elapsed time to determine the rate of linear nail growth. Thus, a weekly or a daily nail growth is conveniently calculated. Measurement is made using a magnifier equipped with a linear scale reaching $0.1 \mathrm{~mm}$ in accuracy. Alternatively, a screw set two-pointed caliper marked the distance from the groove to the proximal nail skinfold. The distance between the points of the caliper is measured to the nearest $0.1 \mathrm{~mm}$ with a micrometer. Circadian variations in body temperature have been reported to influence the linear nail growth rate with a split-image range finder adapted to a trinocular microscope [14]. With such instrumentation, nail growth measurements were claimed to be possible over short intervals.

Nail growth was assessed after indenting the nail surface and measuring the volumetric change of the grooves as they reached the free edge [16]. Erosion was responsible for a $30-50 \%$ reduction in volume [17].

Such methods allow to evaluate the rate of improvement or degradation of the nail condition. 
Fig. 40.2 Nail surface weathering on the proximal part of a nail in the twenty-nail dystrophy. (a) Two-dimensional field image of a nail surface recording. (b) Profilometric presentation
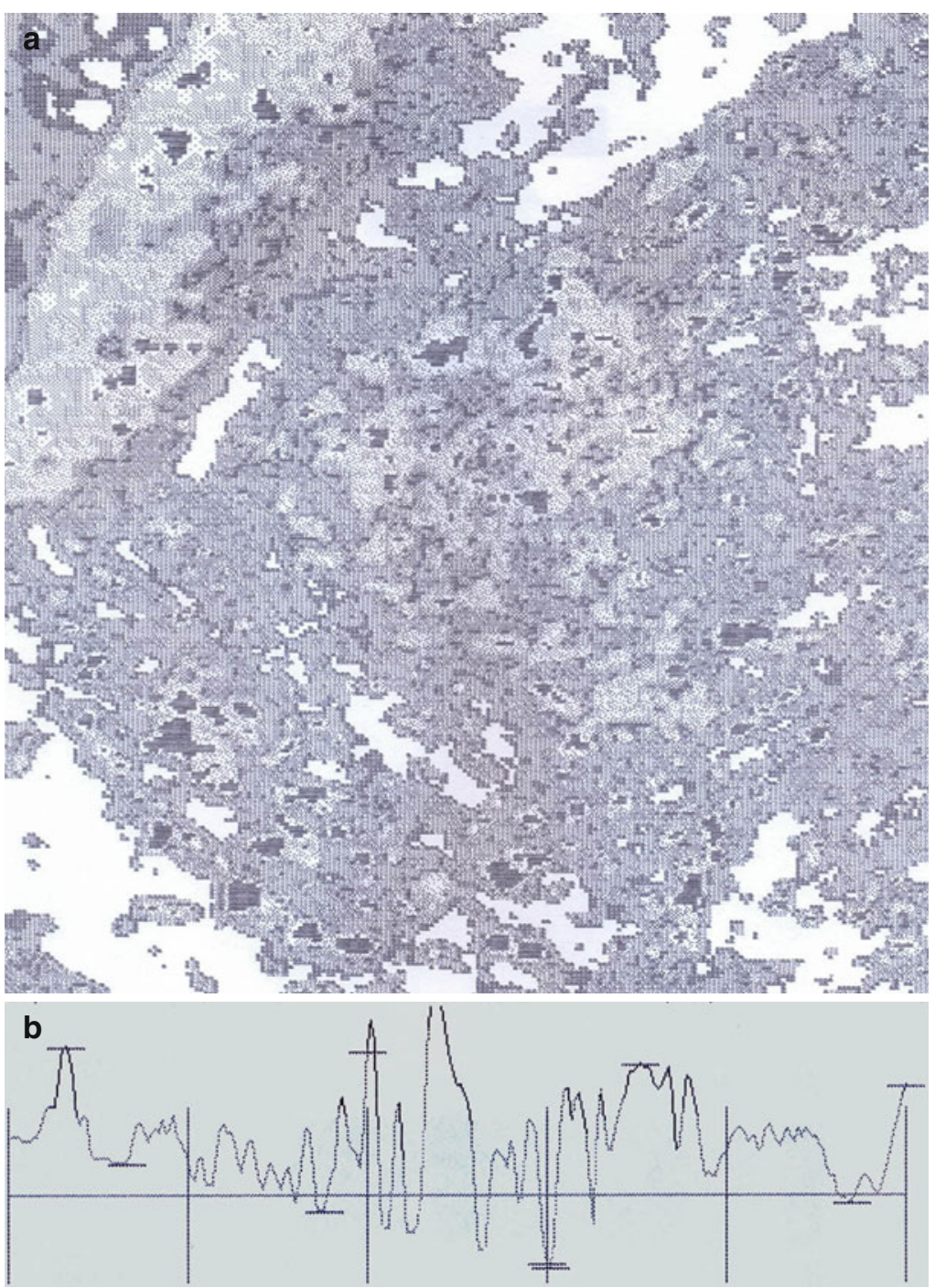

A relevant biological interval should be respected between successive measurements. In this consideration the speed of nail growth must be taken into consideration. Indeed, there is likely interdependence between the disclosed microtopographic changes and variations in the nail growth rate. An example is given by the Beau's lines and beaded nail [7]. Seasonal variations in the nail surface microtopography were reported to vary from insignificant to quite obvious [12].

\subsubsection{Nail Microindentation}

Experimental microindentation allows to assess some mechanical properties of the nail plate following a load application under controlled conditions on a small nail surface. The nail indentation is possibly measured during the test procedure. If any residual plastic deformation persists after releasing the indentation force, the imprint into the nail can be measured using profilometry. 
Fig. 40.3 Nail surface weathering on the distal part of a nail in the twenty-nail dystrophy. (a) Twodimensional field image of a nail surface recording. (b) Profilometric presentation
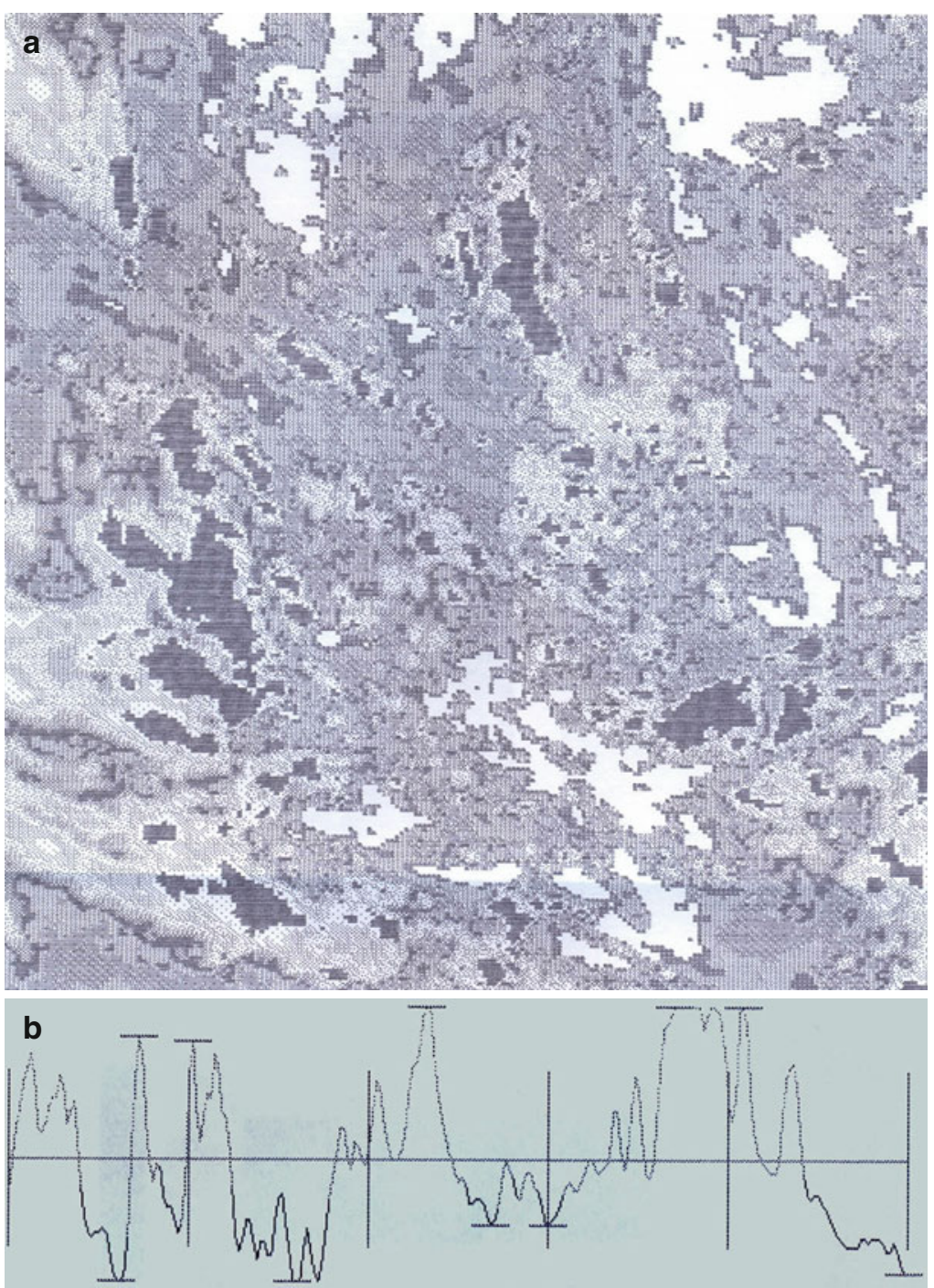

\subsubsection{Nail Sclerometry}

Sclerometry deals with the dynamic assessment of the response of an object during microabrasion. In addition to the classic abrasion parameters, profilometry reveals any groove traced into the nail. The effect of nail hardening products and nail protectors can be tentatively assessed by that way. Similarly, nail softening by xenobiotics or altered health condition can be quantified.

\section{Conclusion}

Objective assessments of the nail surface topography were seldom addressed in the literature. However, the nail microrelief is variably influenced by various physiologic parameters, weathering, external trauma, and pathologic features altering the nail matrix. Some microtopographic alterations are linked to changes in the nail growth rate and in the nail hardness. Presumably some methods developed for the 
skin surface microtopography can be applied to the nail apparatus. These methods should highlight the features of onychochronobiology, thus providing unique information about the physiopathologic processes having altered the nail over the past weeks and months.

Acknowledgments This work was supported by a grant from the "Fonds d'Investissement de la Recherche Scientifique" of the University Hospital of Liège. No other sources of funding were used to assist in the preparation of this manuscript. The authors have no conflicts of interest that are directly relevant to the content of this review. The authors appreciate the excellent secretarial assistance of Mrs. Ida Leclercq and Marie Pugliese.

\section{References}

1. Reich K (2009) Approach to managing patients with nail psoriasis. J Eur Acad Dermatol Venereol 23:S15-S21

2. Rich P, Scher RK (2003) Nail psoriasis severity index: a useful tool for evaluation of nail psoriasis. J Am Acad Dermatol 49:206-212

3. Baran RL (2004) A nail psoriasis severity index. Br J Dermatol 150:568-569

4. Williamson L, Dalbeth N, Dockerty JL et al (2004) Extended report: nail disease in psoriatic arthritis clinically important, potentially treatable and often overlooked. Rheumatology 43:790-794

5. Jiaravuthisan MM, Sasseville D, Vender RB et al (2007) Psoriasis of the nail: anatomy, pathology, clinical presentation, and a review of the literature on therapy. J Am Acad Dermatol 57:1-27
6. Lévêque JL (1999) EEMCO guidance for the assessment of skin topography. J Eur Acad Venereol 12:103-114

7. De Doncker P, Piérard GE (1994) Acquired nail beading in patients receiving itraconazole: an indicator of faster nail growth? A study using optical profilometry. Clin Exp Dermatol 19:404-406

8. Nikkels-Tassoudji N, Piérard-Franchimont C, De Doncker P, Piérard GE (1995) Optical profilometry of nail dystrophies. Dermatology 190:301-304

9. Piérard GE, Piérard-Franchimont C (1996) Fractal microrelief of the skin and nail. Giorn Int Dermatol Ped 8:75-79

10. Piérard-Franchimont C, Piérard GE (1998) Surface image analysis of nail alterations in juvenile pityriasis rubra pilaris. Skin Res Technol 4:34-36

11. Piérard GE, Piérard-Franchimont C (1996) Dynamics of psoriatic trachyonychia during low dose cyclosporin A treatment A pilot study on onychochronobiology using optical profilometry. Dermatology 192:116-119

12. Piérard-Franchimont C, Jebali A, Ezzine N, Letawe C, Piérard GE (1996) Seasonal variations in polymorphic nail surface changes associated with diabetes mellitus. J Eur Acad Dermatol Venereol 7:182-183

13. Piérard-Franchimont C, Piérard GE (2005) Image analysis of the nail surface. In: Serup J, Jemec G, Grove $\mathrm{G}$ (eds) Handbook of non-invasive methods and the skin, 2nd edn. CRC Press, Boca Raton, pp 925-928

14. Orentreich N, Markofsky J, Vogelman JH (1979) The effect of aging on the rate of linear nail growth. J Invest Dermatol 73:120-130

15. Dawber RPR (1970) Fingernail growth in normal and psoriatic subjects. Br J Dermatol 82:454

16. Heikkila H, Stubb S, Kiistala U (1996) Nail growth measurement employing nail indentation - an experimental follow-up study of nail growth in situ. Clin Exp Dermatol 21:96-99

17. De Berker D (1997) Nail growth measurement by nail indentation. Clin Exp Dermatol 22:109-111 